Rosie Doy, Derek Burroughs, John Scott

Emerg Med J 2005;22:279-285. doi: 10.1136/emj.2004.023044

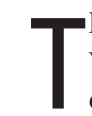

his article has been written with reference to the mental health legislation in England. Variations in this legislation occur in other countries of the UK. Practitioners working in other countries of the UK will require knowledge of the specific legislation for their country. Mental health problems present in between $30 \%$ and $60 \%$ of primary care consultations. ${ }^{1}$ One in six men and one in four women will suffer from a mental illness at some point in their lives. ${ }^{23}$ GPs, for example, find that at least $30 \%$ (or 1.5 days per week) of their working week concerns mental health consultations. For depression alone, prevalence amongst the adult population in the UK varies between 17-71 per thousand for men and from 25-124 per thousand for women.

The standards of the Mental Health National Health Service Framework (MHNSF) aim to support more consistent access and delivery of primary care services including out of hours and non-scheduled care at times of complex need and mental health crisis.

Mental health presentations in primary care are frequently complex and do not always fit easily into diagnostic categories. ${ }^{4}$ This article will specifically consider depression and deliberate self harm; patients with psychoses will be considered in a second paper to follow.

\title{
ARTICLE OBJECTIVES
}

- To clarify definitions of mental disorder

- To consider key aspects of the Mental Health Act (1983) and assessment of valid consent and capacity

- To discuss the recognition of primary survey positive patients and those with complex but not immediately life threatening presentations (primary survey negative patients)

- To describe mental health assessment and differential diagnoses with specific reference to depression and deliberate self harm

- To discuss alternatives to admission, treatment, and options for referral

\section{COMMON SIGNS AND SYMPTOMS, DEFINITIONS, AND THE MENTAL HEALTH ACT}

Just as in other aspects of illness, adequate and accurate description of the signs and symptoms aids communication and patient care. This is especially important in the field of mental health emergencies, where there can be significant difficulty with definitions of mental illness. There is also greater potential for conflict between the individual human right of autonomy and the professional duty of care to act in the patient's best interest.

Patients who are have mental disorder requiring immediate treatment most commonly consent to that treatment or even if they do not consent they may be often treated under "Common Law" in the "patients best interest" (although this has been recently challenged, see below). Approximately $90 \%$ of those admitted to Mental Health Hospitals are admitted as "informal" patients, only $10 \%$ nationally are admitted under a "Section" of the Mental Health Act (MHA), against their will.

Key to the understanding of the MHA is the definitions of mental disorder.

Mental Disorder: ... mental illness, arrested or incomplete development of mind, psychopathic disorder, and any other disorder or disability of mind.

The 4 sub-categories of Mental Disorder are further defined.

See end of article for authors' affiliations

Correspondence to: R Doy, r.doy@uea.ac.uk

\section{Mental Illness:}

There is no legal definition but there are many terms and definitions, see for example JRCALC Clinical Practice Guidelines (June 2004) ${ }^{5}$ or the International Classification of Diseases, version 
10. ${ }^{6}$ Mental illness may be defined as a number of conditions typically involving impairment of an individual's normal cognitive (thinking), emotional, or behavioral functioning. Mental illness may be precipitated by biopsychosocial, biochemical, normal, and traumatic life events, genetic or other factors, including infection or head injury.

The following are some of the potential characteristics of mental illness presentations:

- impairment of intellectual functions shown by a failure of memory, disorientation, difficulties in comprehension, or learning capacity;

- persistent alteration of mood affecting the patient's daily life

- delusional beliefs

- abnormal perceptions (hallucinations) associated with delusional beliefs and thinking patterns

- disordered thinking (cognition), which prevents the patient exercising judgment and perceiving the consequence of their actions

\section{Box 1 Common Signs and Symptoms of Mental} Illness

- Changes in the nature of mood (affect)

- Disorders of perception-hallucinations and illusions

- Disorders of thinking (cognition)-delusions, negative thinking etc

- Patient's appearance and behaviour

- Speech and communication

\section{Severe Mental Impairment:}

... a state of arrested or incomplete development of mind, which includes severe impairment of intelligence and social functioning, and is associated with abnormally aggressive or seriously irresponsible conduct on the part of the person concerned

\section{Mental Impairment:}

... a state of arrested or incomplete development of mind (not amounting to severe mental impairment), which includes significant impairment of intelligence and social functioning and is associated with abnormally aggressive or seriously irresponsible conduct on the part of the person concerned

\section{Psychopathic Disorder:}

... a persistent disorder or disability of mind (whether or not including significant impairment of intelligence), which

\section{Abbreviations}

- ASW: Approved Social Worker

- CRHTT: crisis resolution and home treatment terms

- DSH: deliberate self harm

- MHA: Mental Health Act

- MHNSF: Mental Health National Health Service Framework

- PICU: Psychiatric Intensive Care Unit results in abnormally aggressive or seriously irresponsible conduct on the part of the person concerned

\section{IMPORTANCE OF THE DEFINITIONS}

- The definitions are legal terms, but the diagnosis of a type of Mental Disorder is a matter for clinical judgement.

Use of alcohol and other substances might sometimes cause a Mental Disorder, which is within the scope of the Act, but use of these substances in itself is not within the scope of the Act.

Only a small number of professionals are involved in applying the MHA, principally Approved Social Workers (ASWs), GPs, and doctors approved under Section 12 of the Act (either psychiatrists or others with experience in mental health who have been certified by the Department of Health). Each professional completing a recommendation for detention performs a detailed assessment of the patient's mental state and circumstances. If any one of them feels there is insufficient evidence to recommend detention, the person cannot be kept in hospital.

(Mental health law is currently under review, the draft Mental Health Bill is currently receiving detailed scrutiny (and engendering great debate) prior to enactment in due course. The MHA (1983) therefore is the current mental health legislative guidance.)

In an emergency situation the Police have powers of arrest under section 136 of the MHA. Under section 136 the Police may remove the patient to a place of safety for detention for up to 72 hours. The patient may be detained within that time period by anyone accepting custody from the Police, and a place of safety can include an NHS hospital, Police station, mental health nursing home, residential home for the mentally disordered, Social Services residential accommodation, or any other suitable place if the occupier is willing. It is important to note that Section 136 does NOT include the power to impose treatment without consent. The patient has the right to consult a solicitor in private and to have a person of their choice present.

\section{POSSIBLE ACTIONS FOR TREATMENT OF A PATIENT WITH MENTAL DISORDER}

In situations where a person is suffering from, "Mental Disorder" and refuses intervention for that mental disorder, then the authority for intervention may be the MHA (1983)

The presence of Mental Disorder does not in itself render the individual unable to give valid consent. Valid consent is

Possible actions for treatment of a patient with mental disorder

\begin{tabular}{|c|c|}
\hline Patient gives consent & TREAT \\
\hline $\begin{array}{l}\text { Patient does not consent but is } \longrightarrow \\
\text { incapacitated or unable to give } \\
\text { consent and will comply with treatment } \\
\text { (doctrine of necessity-see below) }\end{array}$ & TREAT \\
\hline $\begin{array}{l}\text { Patient does not consent, and } \longrightarrow \\
\text { will not comply with treatment } \\
\text { suggestions }\end{array}$ & $\begin{array}{l}\text { Consider use of MHA } \\
\text { Consider contacting } \\
\text { Family/carers } \\
\text { GP } \\
\text { Psychiatric team } \\
\text { Police } \\
\text { Mental Health Social Workers }\end{array}$ \\
\hline
\end{tabular}


always "situation specific". An individual who has Mental Disorder may be able to give valid consent for some things, but not for others.

Use of the MHA is strictly defined.

"Informal" patients are either:

- Admitted and treated with their valid consent. Or:

- Incapacitated and unable to give valid consent, so:

Admitted and treated under the Common Law doctrine of:

- "Necessity"

- Acting in the patient's "Best Interests"

- Under a "Duty of Care".

If an incapacitated patient dissents, and the dissent is "persistent and purposeful" - then an assessment should be made for compulsory treatment under the MHA.

A recent ruling by the European Court of Human Rights, $5^{\text {th }}$ October 2004, regarding the "Bournewood" case may have significant impact on the use of Common Law to admit and treat incapacitated adults. At the time of writing the UK Government is considering their position regarding this ruling.

Most importantly, unless the patient has been detained under a relevant section of the MHA (currently section 3), if they have capacity they may legally refuse treatment, even if they are suffering from a mental disorder. Even if detained under section 3, only treatment for the mental disorder can be legally imposed in a patient with capacity. Treatment for a physical disorder which is not associated with the mental illness cannot be imposed under these circumstances.

\section{ASSESSMENT OF VALID CONSENT}

The concepts of "valid consent" and "Common Law" are important in this area of practice. It is an underlying principle of medical care that consent should always be sought before any intervention is commenced. Failure to correctly determine the patient's ability to give valid consent may lead to charges of assault, or battery, or worse. However, there is often a difficult conflict between the patient's right to determine their own treatment and the professional responsibility to act "in the patient's best interests". Failure to intervene and care for a patient who cannot give valid consent may lead to charges of negligence.

A good understanding of the Law and how to apply it to practice empowers not only the practitioner, but also the patients with whom we work.

How then do we determine where our legal authority comes from when working with patients? Initially we must attempt to gain our authority from the patient, via their valid consent. Valid consent comprises three components. The absence of any one component will render the consent invalid.

There are three components of valid consent:

\section{Box 2 Components of valid consent:}

- Competence/capacity

- Disclosure of Information

- Voluntariness

1. Is the patient competent, or, does the patient have 'capacity' to consent? Competence and capacity are used interchangeably in this context.
Assessment of a patient's capacity to make a decision about their own health care, is a matter of clinical judgement, guided by current professional practice and subject to legal requirements. It is the personal responsibility of any health care professional proposing treatment to determine the patient has capacity to give valid consent.

An individual is presumed to have capacity to decide on a particular treatment. This is termed a "positive presumption of capacity".

\section{The Capacity test:}

Is the person able to take in and retain information material to the decision, especially as to the likely consequences of having, or not having the treatment?

Or:

Is the patient unable to weigh the information in the balance, as part of the process of arriving at the decision?

It is important to remember that capacity will be affected by common trauma situations such as, shock, pain, or fear. 2. The second component of valid consent is "Disclosure of Information"

We must give the patient adequate information so that they can make an "informed choice". The law requires that we inform the patient of the "broad terms of the nature of the procedure". We clearly cannot tell them all the details, but we must ensure they have adequate information on which to base a decision.

3. The third component of valid consent is "voluntariness". We must ensure that neither we, nor anyone else, put undue pressure on the patient to comply with our wishes to intervene.

There will be many instances where care or treatment is needed, it is obvious that the patient is an adult, but is unable to give valid consent. They may be unconscious, traumatised or perhaps have ongoing dementia, or a mental disorder.

Given that no one else can give valid consent for another adult, "Common Law" comes into effect. If the adult patient does not meet the criteria for valid consent then the Common Law doctrine of, "necessity" applies. We must be able to demonstrate that our interventions are "necessary", necessary to save life or "prevent deterioration" of the situation.

We must be able to demonstrate that our interventions are in "The patient's best interests" and be able to demonstrate we have a, "Duty of Care" to the patient. If we can demonstrate that all three criteria are present, and we are satisfied that we cannot, for whatever reasons, obtain valid consent from the adult patient, then Common Law empowers us, and gives us the authority for intervention. Indeed in these situations, if we do not intervene, then we may be held to be "negligent" in Law.

The government has recently published a "Capacity Bill" in which they intend to move some of these powers from "Common Law" to "Statute Law". They also intend to make other provisions for the care and treatment of incapacitated adults. This is unlikely to become Law before 2006 .

\section{PRIMARY SURVEY}

A mental illness may cause a patient to take an overdose or injure themselves in such a way that they develop immediately life threatening ABCD problems. These problems are covered in other articles. 
An immediately life threatening situation that may arise is where the patient is saying they are going to kill themselves or harm others but will not comply with treatment. The reaction to this problem will depend on a large number of variables including your assessment of the problem and the help available.

282 Enlisting the support of family and carers is often the simplest and best way to resolve such conflicts. However, if this does not work you will have to call for assistance. This may be the patient's own primary care team or the mental health team. In extreme situations where you judge that there is an immediate threat to the wellbeing of the patient or others you should call the police.

The MHA, in Section 63, states that the detained (or 'sectioned') patient's consent is not needed for medical treatment for mental disorder (when this is under the direction of the Responsible Medical Officer.) This is detailed as being:

(a) "immediately necessary to save the patient's life; or

(b) which (not being irreversible) is immediately necessary to prevent a serious deterioration of his condition; or

(c) which (not being irreversible or hazardous) is immediately necessary to alleviate serious suffering by the patient; or

(d) which (not being irreversible or hazardous) is immediately necessary and represents the minimum interference necessary to prevent the patient from behaving violently or being a danger to himself or to others."

However, the MHA does not necessarily permit the compulsory treatment of a physical disorder in a patient who is not consenting.

\section{Box 3: Primary Survey}

- Is the patient threatening to kill or seriously harm themselves?

- Is the patient a risk or danger to themselves or to others?

- Is the patient through lack of appreciation of the consequences likely to come to harm?

- Is the patient, through lack of capacity, vulnerable to abuse from others?

- Has the patient become acutely confused?

\section{SECONDARY SURVEY}

If it is obvious that the patient is going to have to be assessed by another professional then only a brief evaluation will be required. However, the following steps need to be taken to ensure the patient is suffering from a mental illness and not a physical disorder. Acute infections, intoxications, drug withdrawal syndromes, diabetes, and neurological conditions are common physical conditions that may present with symptoms of mental illness/disorder. An acute confusional state may present very rapidly, especially in older patients, this may result from chest or urinary tract infections as well as in the older person who has experienced recent life change, or who is suffering from dementia.

\section{践 Tip}

Ensure the patient is not suffering from a physical disorder

\section{Box 4 Mental Health Assessment}

- Presenting problem

- History of presenting problem

- Previous medical history

- Drug history / allergies/ concordance

- Past psychiatric history

- Previous contact with services

- Carer information

- Brief physical examination (vital signs including temperature if possible)

- Appearance

- Behaviour

- Cognition, concentration, and attention span

- Ideation, i.e. beliefs and level of insight

- Energy and motivation

- (free) choice and volition

- Risk- to self, to others, of deliberate self harm (DSH), or neglect

- Impulsivity

- Co-morbidity- drugs/ alcohol

- Social factors and support including family history

- Precipitating factors

- Coping strategies

Box 4 summarises the assessment of a patient with psychiatric symptoms.

Effective and sensitive mental health assessment involves a person-centered consultation style. A therapeutic relationship is central to this, as is gaining the patient's trust and showing the patient that you recognise their distress and experience. Some key principles for the mental health interview are identified in Box 5 below. Consultation skills that improve identification of emotional distress include frequent eye contact, relaxed posture, use of open questions at the beginning of the consultation, use of minimal verbal prompts while actively listening, and avoiding giving information too early in the consultation

\section{Box 5 The Mental Health Patient-Centered}

\section{Interview}

- Actively listen and be alert to your observations

- Allow the patient to explain the problem in their own words

- Be non-judgemental

- Use focusing techniques to enable the patient to gain selfcontrol

- Seek clarification use of paraphrasing, reflection, and summarising

- Sharing impressions

- Use of silence

\section{珨 Pitfall}

Jumping to conclusions

\section{諬 Tip}

Talk to the family and get their viewpoint 
At the end of the assessment you should be able to judge if the patient has

- A physical disorder

- A disorder of mood (commonly depression)

- Acute anxiety or panic

- Acute confusion

- A psychotic disorder

- Or is a threat to themselves or others

The actions required will depend on your assessment of the severity of the condition; the options are discussed in the section on treatment and referral.

\section{THE DEPRESSED PATIENT}

Depression is the most common mental disorder in primary care. The term depression covers a range of mental health diagnoses and problems. These problems are distinguished by lowered mood and a loss or decrease of interest and pleasure in daily life and experiences. Additionally, there are disorders of thinking, problem-solving, and behavioural and physiological symptoms. ${ }^{7}$ It is not easy to discriminate between normal mood variations, dysthymia and cyclothymic (see Box 7) episodes and mild to moderate clinical depression. Box 6 lists the diagnostic criteria for severe depression.

\section{Box 6 Recognition and Classification of Severe} (Major) Clinical Depression

At least five of the following symptoms are consistently experienced by the client on a daily basis over a 2 week period:

- Persistent sad mood

- Loss of interest or pleasure in activities that were once enjoyed

- Significant weight loss or gain without dieting, increased or reduced appetite

- Insomnia or hypersomnia

- Psychomotor agitation or retardation

- Fatigue or listlessness; loss of energy

- Feelings of worthlessness and inappropriate guilt

- Reduced ability to concentrate, make decisions or think

- Recurrent thoughts of death or suicidal ideation
Box 7 Differential Diagnoses-Mood Disorders

- Clinical Depression

- Severe

- Moderate

- Mild

- Bipolar Disorder (Manic Depression)

- Dysthymic episodes- Chronic low grade depression (for at least 2 years)

- Cyclothymia-cycling variations in mood; much less extreme than in Manic Depression

- Seasonal Affective Disorder

- Post-Natal Depression

- Psychotic depression

\section{SUICIDE RISK}

Patients with mental illness have an increased risk of suicide. In fact each year people with depression account for two thirds of all deaths from suicide nationally. Risk assessment tools and rating scales can be very helpful, for example, the Suicide and Self-Harm Risk Assessment Scale:

\section{Suicide risk \& Self-Harm Risk Assessment Scale}

\begin{tabular}{ll}
\hline Sex male & 1 \\
Age $<19$ or $>45$ years & 1 \\
Depression/hopelessness & 1 \\
Previous attempts & 1 \\
Excessive alcohol/drugs & 1 \\
Rational thinking (loss of) & 1 \\
Separated widowed divorced & 1 \\
Organised or serious attempt & 1 \\
No social support & 1 \\
Stated future intent & 1
\end{tabular}

Score $\leqslant 3$ low risk, $3-6$ medium risk, $>6$ high risk. This is a guide only and should not be used to replace clinical judgement. (See JRCALC guidelines A6).

\section{TREATMENT AND REFERRAL}

Prior to the MHNSF the traditional management of the at risk suicidal patient was by admission to an acute mental

\section{Box 8 Additional Risk Factors for Suicide}

- Up to 4 weeks following discharge from services

- Recent self harm; history of violent self harm (half of those who commit suicide will have self-harmed in the past $^{12}{ }^{13}$ )

- Depression- as mood lifts

- Choice of method

- Young Asian women

- Some occupations and social groups- dentists, doctors; farmers, unemployed, homeless or living alone, students, divorced, separated or widowed (men)

- Relationship problems

- Chronic illness: including HIV/AIDS, cancer, diabetes, post stroke especially when communication centres affected, Parkinson's Disease, Huntington's Disease, and Alzheimer's Disease (where some insight remains)

- Care givers without adequate social support/ financesespecially carers of those who are severely cognitively impaired

- Chronic pain suicide. A number of patient's who successful commit suicide immediately preceding period. At least $30 \%$ see their GP in the 4 weeks prior to their deaths. ${ }^{8}$ Improving the recognition of severe depression and its treatment has been the focus of long term data shows little sustained difference.

It is often helpful to support the patient in 'telling their story' - what a typical day is like, what makes it better or worse and listening carefully not only to 'what' they say but 'how' they express their narrative.

The patient with depression may be at risk of suicide. 


\section{Tip}

Ask about suicide. Gentle questioning including:

'Do you feel that you don't want to go on any more?'

'Have you thought about what you would do?'

'Have you developed a plan?'

\section{Pitfall}

Believing the patient when they say they will not try to kill or harm themselves

health unit. In non-scheduled and out of hours care, this is problematic due to bed shortages, high acute in-patient bed occupancy (often in excess of 100\%) and implementing MHA processes for detaining at risk patients unwilling to agree to admission. Currently there is growth in managing these patients in the community and many Crisis Resolution and Home Treatment Teams (CRHTTs) have been developed to offer intensive community-based interventions in the patient's own home (MHNSF target of 335 CRHTTs by this year). Where such teams exist, a referral both 'in hours' and 'out of hours' to the local CRHTT should be made. The CRHTT will undertake a comprehensive mental health and risk assessment. As appropriate, a treatment, support, or monitoring package will be implemented.

Other alternatives to admission that may be available include acute day hospital care, crisis beds, the Community Mental Health team and the Primary Care Gateway (Link) Worker or 24 hour and weekend help and support lines. Services are, however, very variable from PCT to PCT. In some areas Psychiatric Intensive Care Unit (PICU) beds may be available for urgent admission, in others assertive outreach or assertive community treatment or other intensive case management services may be available.

\section{Box 9 Treatment Options}

1. Admission to Hospital:

- severe depression especially when suicide has been attempted or serious suicide ideation is present and the patient requires close or constant observation

2. ED and EAU Mental Health Assessment Liaison Team: - full psychosocial assessment (as recommended by NICE, 2004) and referral or brief interventions offered as appropriate; may offer specific alcohol and substance misuse service

3. Home Treatment and Crisis Resolution via local mental health $\mathrm{OOH}$ services:

- full psychosocial assessment and service provision for the patient with mental illness who can be supported/ treated at home without admission

4. Medication:

- Fluoxetine or Paroxetine may be prescribed for moderate to severe depression; Risperidone for psychosis and agitated confusion; Chlorpromazine and Haloperidol for psychosis, confusion, and behavioural disorders

5. Talking Treatments:

- Referral to mental health team/GP

NOT appropriate for people with severe depression

Box 10 Principles of Effective Shared and $\mathrm{OOH}$

\section{Care:}

- Develop crisis care plans with agreed contact points for known patients and ensure they are available to $\mathrm{OOH}$ services

- Agree $\mathrm{OOH}$ arrangements and ensure they are available to all relevant team members including locums

- Clarify criteria for referral and discharge between primary and secondary care; between $\mathrm{OOH}$ and mental health crisis teams

- Develop mental health registers in primary care

- Joint inter-professional and multi- agency education, e.g. suicide assessment and risk management

- Consistent and regular communication with regular liaison meetings; systematic review of shared care and complex patients

- Robust record-keeping

- Rotations between $\mathrm{OOH}$ services

- Regular audit

Liaison nurses in emergency and MAU departments of acute general hospitals are also useful resources for both inpatient and primary care practitioners.

\section{DIFFERENTIATING BETWEEN SUICIDE AND DELIBERATE SELF-HARM (DSH)}

The MHNSF indicates that overall the rate of suicide is dropping. ${ }^{9}$ Men are three times more likely than women to commit suicide; women are three to four times more likely to present with deliberate self-harm by overdosing, cutting, or other means. ${ }^{10}$ Whilst suicide is rare, the average PCT (population of 100,000) would have about 10 suicides per annum. The term deliberate self-harm (DSH) indicates that the person hurts themselves, either to signal distress, in crisis and where coping strategies are limited, and to release/ manage overwhelming feelings. ${ }^{11}$ So, whilst there may be no 'intention' to kill themselves the person who is self-harming does increase the risk of death with each occasion of this behaviour. NICE identify that there are 150,000 attendances at A\&E each year resulting from DSH therefore being one of the top five causes of acute medical admission..$^{12}$

Box 11 Why does the client self-harm? Four main themes regarding motivation emerge from experiential and empirical research evidence.

1. handling and expressing overwhelming feelings

2. escaping numbness/ unreality and confirming one's existence

3. obtaining or maintaining a sense of control

4. continuing past abusive patterns

(Adapted from Doy, 2003)

\section{Pitfall}

Diminishing the behaviour and judging it as (only) attentionseeking

\section{咸 Tip}

Behaviour always has a meaning- we often do not appreciate what it means for the person 
At times of crisis it is easy to disempower the person who has self-harmed by dismissing their often frustrating and repetitive behaviour as 'manipulative'. Such clients are often overwhelmed and chaotic with limited coping strategies, low self-esteem, and perceptions of a lack of control and safety in their lives.

\section{蘶Tip}

Practitioners who have first contact with people who have intentionally self- harmed should:

- undertake a psychosocial and needs assessment at triage or initial assessment

- undertake risk assessment

- assume capacity unless there is evidence to the contrary

- offer full information and seek valid consent

- recognise the distress associated with deliberate self-harm and treat the person with respect

- Make appropriate referrals

If the client is not primary survey positive use of patientcentered consultation skills (see Box 5) and suitable referral and signposting will be appropriate.

\section{Box 12 Relevant Services and help lines}

- Bristol Crisis Line (National part-time help line)

- Community Mental Health Team

- CRHTT

- Mental Health Liaison Nurses- ED; MAU

- National Self-Harm Network

- Primary Care Gateway (Link) Workers

It is important to provide the patient with alternatives to self-harming including help-line contact and for pre-hospital workers to consider referral for psycho-therapy. Many social care or voluntary agencies may be effective in supporting the patient with relationship, accommodation, financial, substance misuse, abuse, and violence issues.

As can be seen, mental health and its management in community settings is complex. The key challenges include developing competence in assessment and risk assessment, in clarifying roles and services in the $\mathrm{OOH} /$ emergency contexts and drawing up clear and agreed guidelines and communication channels.

\section{SUMMARY}

This paper has considered definitions of mental disorder and key aspects of the MHA with particular emphasis on valid consent. Mental illness, its assessment and referral pathways within the pre-hospital setting have been considered, with particular emphasis on depression and deliberate self harm. A future article will consider psychoses, personality disorders and dementia in more detail.

Mental illness is hugely debilitating for many patients; taking huge toll on their families and carers. Detailed assessment and early referral can help in the early diagnosis of mental disorder (leading to greatly enhanced prognosis); and to early intervention in relapse or crisis situations. The development of mental health CRHTTs and ED liaison teams, the advent of the Primary Care Gateway (Link) Worker and of the Emergency Care Practitioner is supporting enhanced referral and greater coherence within the patient pathway. It is hoped that this paper will enable pre-hospital care practitioners to identify and assess the person with depression with greater confidence and to be aware of additional referral pathways available for these patients.

\section{Authors' affiliations}

R Doy, D Burroughs, School of Nursing and Midwifery, University of East Anglia, UK

J Scott, East Anglian Ambulance NHS Trust, UK

\section{FURTHER READING}

1 Department of Health. Reference Guide to Consent for Examination or Treatment. London: DH, 2001.

2 Hatton C, Blackwood R. In: Lecture Notes on Clinical Skills, $4^{\text {th }}$ edn., Oxford: Blackwell Science, (Chapter 6) 2003.

3 Joint Royal Colleges Ambulance Liaison Committee, 2004: Clinical Practice Guidelines version 3. 0, University of Warwick/JRCALC available from http://www.nelh.nhs.uk/emergency.

4 Mynors-Wallis L, Moore M, Maguire J, et al. Shared Care in Mental Health. Oxford: Oxford University Press, 2002.

5 National Institute of Clinical Excellence. The NICE website contains clinical guidelines for depression and anxiety, also for schizophrenia and self-harm www.nice.org.uk.

6 The Mental Health Act 1983: Guidance for general practitioners: medical examinations.

7 Medical recommendations under the Act, BMA 1999

8 World Health Organisation, 1992 ICD-10 Classification of mental and behavioural disorders. Geneva: WHO.

\section{REFERENCES}

1 MACA. First National Survey of Mental Health in Primary Care, MJM: Pfizer 1999.

2 Department of Health. Modern Standards and Service Models: Mental Health. London: NHSE, 1999.

3 National Institute of Clinical Excellence, 2004a: Depression: the management of depression in primary and secondary care. http://www.nice.org.uk (accessed 27 Feb 2005)

4 Mynors-Wallis L, Moore M, Maguire J, et al. Shared Care in Mental Health. Oxford: Oxford University Press, 2002.

5 Joint Royal Colleges Ambulance Liaison Committee. Clinical Practice Guidelines version 3.0, University of Warwick/ASA/JRCALC 2004.

6 World Health Organisation. ICD-10 Classification of mental and behavioural disorders. Geneva: WHO, 1992.

7 World Health Organisation. WHO Guide to Mental Health in Primary Care. London: Royal Society of Medicine Ltd, 2000.

8 Evans J. Suicide, Deliberate self-harm, and severe depressive illness. In: Elder A, Holmes J, eds. Mental Health in Primary Care. Oxford: OUP, 2002.

9 Department of Health. National Suicide Prevention Strategy for England. London: DH, 2002

10 Doy R. Women and Deliberate Self-Harm. In: Boswell G, Poland F, eds. Women's Minds, Women's bodies: An Interdisciplinary Approach to Women's Health. Basingstoke: Palgrave Macmillan, 2003.

11 Burstow B. Radical Feminist Therapy: Working in the Context of Violence. London: Sage, 1992.

12 National Institute of Clinical Excellence, 2004b: Self-harm: The short-term physical and psychological management and secondary prevention of selfharm in primary and secondary care. http://www.nice.org.uk (last accessed 28 July).

13 National Institute of Clinical Excellence 2004c: Scope: Self-harm: The shortterm physical and psychological management and secondary prevention of self-harm in primary and secondary care. http://www.nice.org.uk (last accessed 28 July). 